\title{
Erratum to: Synthesis, Structure, and Biological Activity of Palladium(II) Complexes with Some 1- and 2-Substituted Tetrazole Ligands
}

\author{
A. A. Batyrenko ${ }^{a}$, O. V. Mikolaichuk ${ }^{b}$, G. K. Ovsepyan ${ }^{b}$, A. V. Protas $^{c}$, I. V. Kornyakov ${ }^{b, d}$, \\ E. V. Lider ${ }^{e}$, Yu. A. Eremina ${ }^{e}$, T. S. Khlebnikova ${ }^{f}$, F. A. Lakhvich ${ }^{f}$, and R. E. Trifonov ${ }^{a, *}$ \\ ${ }^{a}$ St. Petersburg State Institute of Technology (Technical University), St. Petersburg, 190013 Russia \\ ${ }^{b}$ St. Petersburg State University, St. Petersburg, 199034 Russia \\ ${ }^{c}$ Pavlov First St. Petersburg State Medical University, St. Petersburg, 197022 Russia \\ ${ }^{d}$ Kola Scientific Center of the Russian Academy of Sciences, Apatity, 184209 Russia \\ ${ }^{e}$ A. V. Nikolaev Institute of Inorganic Chemistry, Siberian Branch of the Russian Academy of Sciences, \\ Novosibirsk, 630090 Russia \\ ${ }^{f}$ Institute of Bioorganic Chemistry, National Academy of Sciences of Belarus, Minsk, 220141 Belarus \\ *e-mail: rost_trifonov@mail.ru
}

Received June 16, 2021; revised June 16, 2021; accepted June 22, 2021

DOI: $10.1134 / \mathrm{S} 1070363221060426$

The affilation of E. V. Lider should read as "e": A. V. Nikolaev Institute of Inorganic Chemistry, Siberian Branch of the Russian Academy of Sciences, Novosibirsk, 630090 Russia

The original article can be found online at https://doi.org/10.1134/S1070363221040149 